\title{
The role of brachytherapy in the management of brain metostases: a systematic review
}

\author{
Bhargava Chitti, BS!, Sharad Goyal, MD!, Jonathan H. Sherman, MD², Anthony Caputy, MD², Mehrdad Sarfaraz, PhDl \\ Gizem Cifter, PhD!', Hamid Aghdam, MS!, Yvan James Rao, MDl \\ IRadiation Oncology, George Washington University School of Medicine and Health Sciences, United States, ${ }^{2}$ Neurosurgery, George \\ Washington University School of Medicine and Health Sciences, United States
}

\begin{abstract}
Purpose: Brain metastases have a highly variable prognosis depending on the primary tumor and associated prognostic factors. Standard of care for patients with these tumors includes craniotomy, stereotactic radiosurgery (SRS), or whole brain radiotherapy (WBRT) for patients with brain metastases. Brachytherapy shows great promise as a therapy for brain metastases, but its role has not been sufficiently explored in the current literature.

Material and methods: The PubMed, Cochrane, and Scopus databases were searched using a combination of search terms and synonyms for brachytherapy, brain neoplasms, and brain metastases, for articles published between January $1^{\text {st }}, 1990$ and January $1^{\text {st }}, 2018$. Of the 596 articles initially identified, 37 met the inclusion criteria, of which 14 were review articles, while the remaining 23 papers with detailing individual studies were fully analyzed.

Results: Most data focused on ${ }^{125}$ I and suggested that it offers rates of local control and overall survival comparable to standard of care modalities such as SRS. However, radiation necrosis and regional recurrence were often high with this isotope. Studies using photon radiosurgery modality of brachytherapy have also been completed, resulting superior regional control as compared to SRS, but worse local control and higher rates of radiation necrosis than ${ }^{125} \mathrm{I}$. More recently, studies using the ${ }^{131} \mathrm{Cs}$ for brachytherapy offered similar local control and survival benefits to ${ }^{125} \mathrm{I}$, with low rates of radiation necrosis.

Conclusions: For a variety of reasons including absence of physician expertise in brachytherapy, lack of published data on treatment outcomes, and rates of radiation necrosis, brachytherapy is not presently a part of standard paradigm for brain metastases. However, our review indicates brachytherapy as a modality that offers excellent local control and quality of life, and suggested that its use should be further studied.
\end{abstract}

J Contemp Brachytherapy 2020; 12, 1: 67-83 DOI: https://doi.org/10.5114/jcb.2020.93543

Key words: brain metastases, brachytherapy, radiation therapy.

\section{Purpose}

There are 170,000-200,000 new cases of brain metastases diagnosed each year, and $20-40 \%$ of cancer patients will develop brain metastases [1,2]. Brain metastases are especially important in the context of more effective cytotoxic, biologic, and immunologic systemic therapy, which have afforded patients longer intervals prior to developing brain metastases in passing years. This makes surveillance and management of intracranial disease increasingly important. Prognosis of patients with brain metastases are highly variable, based on the primary tumor and associated prognostic factors. Using the graded prognostic assessment (GPA) index, which divides patients into 4 tiers based on various clinical prognostic factors, median overall survival can range from 2.79 to 25.30 months [3].

The clinical management of single metastases with craniotomy and/or stereotactic radiation is well estab- lished. Level 1 evidence supports the use of stereotactic radiosurgery (SRS) alone, whole brain radiation therapy (WBRT) alone, or surgery in combination with SRS or WBRT in patients with single or multiple brain metastases (MBM) [4]. Choosing an appropriate treatment for a patient with brain metastases is quite personalized and requires close collaboration between neurosurgeons, radiation oncologists, and oncologists, in an effort to maximize and balance both survival and quality of life.

Despite its many benefits, brachytherapy is a relatively uncommon modality for the treatment of brain metastases. This treatment technique involves the implantation of radioactive isotopes at the time of tumor resection for brain metastases. Since brain metastases tend to occur relatively superficially in the brain, often in the grey-white matter interface, and are frequently surgically resected, patients with brain metastases may be ideal candidates
Address for correspondence: Sharad Goyal, MD, Radiation Oncology, George Washington University School of Medicine and Health Sciences, 2150 Pennsylvania Avenue, NW, DC Level, Washington, DC 20037, United States, phone: +1 202715 5097, fax: +1 202 715 5136, « e-mail: shgoyal@mfa.gwu.edu
Received: 22.10.2019

Accepted: 17.02 .2020

Published: 28.02.2020 
for brachytherapy. Through this technique, one can deliver a highly conformal dose of radiation, with a rapid dose fall-off and the ability to spare surrounding normal brain tissue. The American College of Radiology (ACR) appropriateness criteria for brain metastases describes that despite similar control rates to radiosurgery, brachytherapy is rarely used because it is an invasive procedure requiring hospitalization [5]. Other reasons that may limit the usage of brachytherapy in the management of brain metastases is a rate of radiation necrosis, absence of neurosurgeons' or radiation oncologists' experience, and a relative lack of published data on treatment outcomes, comparing to other modalities for brain metastases.

Brachytherapy for brain tumors was first used as early as 1936, by Dr. W.O. Lodge, who implanted radon seeds in the brain of a patient who was suffering from a pituitary mass that had induced amenorrhea and vision loss [6]. The implant shrunk the tumor and restored the patients' vision rapidly. Since then, ${ }^{125} \mathrm{I}$ became the most frequently used brachytherapy isotope in the treatment of brain tumors, with the first treatment of brain metastases using brachytherapy in 1979 by Prados and colleagues [7]. Subsequently, other studies have been done evaluating the use of intraoperative photon radiation (photon radiosurgery - PRS) as well as other isotopes such as ${ }^{131} \mathrm{Cs}$ $[8,9,10,11,12,13]$. In particular, ${ }^{131} \mathrm{Cs}$ is a promising new isotope for the use in brachytherapy explored by Wernicke and colleagues in a series of studies on local resection followed by implantation of ${ }^{131} \mathrm{Cs}$ seeds in patients with brain metastases $[10,11,12,13]$.

The use of new brachytherapy modalities such as ${ }^{131} \mathrm{Cs}$ brachytherapy may address some of the issues that have limited implementation of brachytherapy in the past. Therefore, the purpose of this paper was to provide a comprehensive summary of the literature on treatment of brain metastases with brachytherapy.

\section{Material and methods}

This systematic review was conducted in accordance with the preferred reporting items for systematic reviews and meta-analyses (PRISMA) guidelines [14]. A literature search of PubMed, Cochrane, and Scopus was conducted by two authors (B.C. and S.G.) using combinations of search terms and synonyms for brachytherapy, brain metastases, radiation, and published between January 1, 1990 and January 1, 2018. The search terms utilized in PubMed included: 1. "Brachytherapy" [Mesh] AND "Brain neoplasms"[Mesh]; 2. "Brachytherapy" [Mesh] AND "Brain neoplasms" [Mesh] and "Neoplasm metastasis" [Mesh]; 3. "Brachytherapy" [Mesh] and "Brain" [Mesh]. The search terms utilized in Scopus were "Brachytherapy" AND "brain" AND "secondary OR metastases OR metastasis" AND NOT "DBCOLL (medl)". The search terms utilized in Cochrane were as follows: \#1: "Brachytherapy [Mesh]"; \#2: “\#1 and brain"; \#3: "Brachytherapy and brain and (secondary or metastases or metastasis," \#4, "\#2, or \#3". In PubMed, Scopus, and Cochrane, we also utilized search terms "iridium radioisotopes" AND "intracranial neoplasm" to assess studies utilizing the ${ }^{192}$ Ir isotope. Additional manual searches in reference li- sts of the relevant articles were also conducted. Studies in non-English languages, duplicate articles, or studies involving animals were excluded. Papers were identified $(n=596)$, from which titles and abstracts were examined to eliminate studies without evidence-based data such as case reports, dosimetry studies, cost-effectiveness studies, comments/responses, reviews, stand-alone abstracts, and studies of primary brain tumors and of pediatric brain tumors. All remaining articles were screened carefully; clinical trials, large observational studies, and studies focusing on brachytherapy in patients with brain metastases received priority in the selection process. Bibliographies of these studies were searched for other relevant studies. Initially, 37 articles were identified, and review articles were excluded $(n=14)$. Of these, the most relevant 23 articles were selected for inclusion (Figure 1).

The resulting papers were reviewed by a multi-disciplinary team composed of medical physicists, neurosurgeons, and radiation oncologists. Critical issues were identified, and key findings from the current literature were summarized in this report. In particular, the clinical characteristics of patients used in the studies, and treatment factors such as radiation isotope (Table 1), radiation dose, and implant volume were recorded from each of the studies $[15,16]$. Outcome variables such as local control, rate of distant recurrence, overall survival, and treatment toxicity were also tabulated and reported. Definitions for local control and distant recurrence were tabulated as per definitions provided in individual papers. However, in general, local control refers to restriction of disease to the area immediately surrounding the resection cavity, while distant recurrence defines disease recurring or progressing outside the immediate area of the resection cavity. A notable exception included studies by Wernicke et al. and Pham et al. who reported $100 \%$ rate of local control, but some instances of regional recurrence defined as dural-based enhancement were $>5 \mathrm{~mm}$ from the resection cavity $[10,11,12,13]$. Summative assessments of treatment efficacy and toxicity were completed based on radioisotopes and brachytherapy techniques used in various studies. A statistical meta-analysis was not attempted due heterogeneity of studies and brachytherapy treatment techniques.

\section{Results}

\section{Iodine-125}

In the literature, most data on treatment of brain metastases with brachytherapy implement the use of ${ }^{125} \mathrm{I}$ isotope $[8,17,18,19,20,21,22,23,24,25,26,27,28,29,30,31,32]$. The largest studies performed in this area include those by Raleigh et al., Ostertag et al., Petr et al., and Ruge et al. $[21,22,23,29,30,31]$. Raleigh et al. conducted a retrospective review featuring 95 patients with 105 brain metastases, treated between 1997 and 2013 with permanent implants, to assess treatment options for patients with recurrent or large brain metastases (Table 2). In regards to location, 32 tumors were located in the frontal lobe, 26 in the parietal lobe, 17 in the occipital lobe, 13 in the cerebellum, 94 in the cerebral/cerebellar convexity, 20 in 


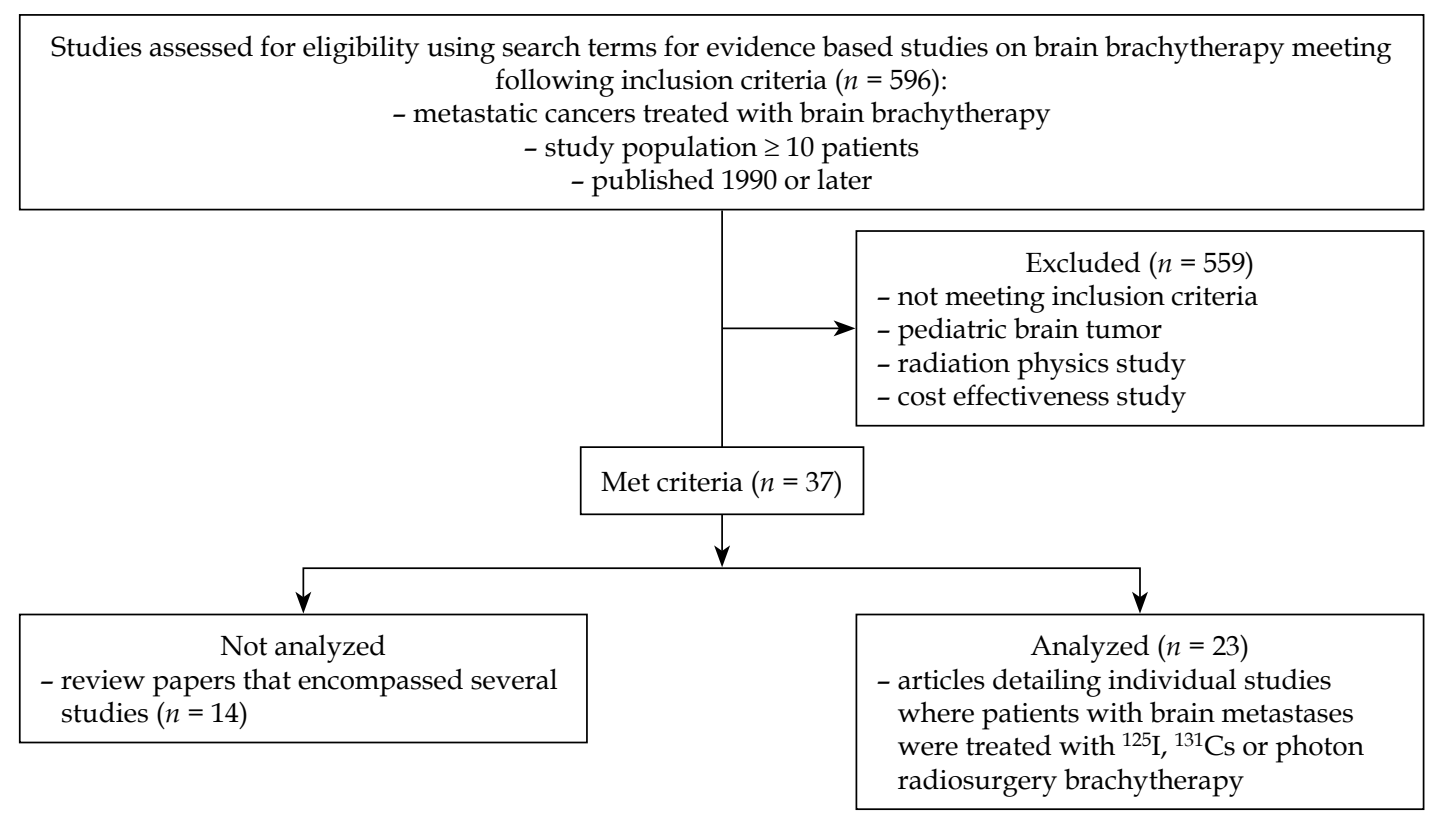

Fig. 1. Consort diagram for patient eligibility, per PRISMA [14]

the periventricular region, and 20 in the lobar tip. Primary tumors included 36 lung carcinomas, 26 melanomas, 22 breast tumors, and 11 tumors in other categories (Table 3). All patients received MRI, followed by a craniotomy with resection of their tumor, and implantation of permanent ${ }^{125} \mathrm{I}$ seeds in the resection cavity. Median number of seeds implanted per cavity was 28 , and median radioactivity per seed was $0.28 \mathrm{mCi}$. They reported $90 \%$ of crude local control rate and distant recurrence rate of $43 \%$ at median follow-up of 4.4 months (Table 4 ). Median overall survival was 12 months, and median Karnofsky Performance Score (KPS) was 80 (range, 5090 months) (Table 5). Their overall risk of necrosis was $15 \%(p<0.001)$, with notable increase in patients with a history of prior SRS $(p<0.05)$ (Table 6). Based on their results, they concluded that ${ }^{125} \mathrm{I}$ seed brachytherapy was an effective strategy for local control of brain metastases. They also noted that volumetric parameters (e.g. metas- tasis or cavity volume, or rate of cavity remodeling) did not influence odds of radiation necrosis or local control. Ostertag et al. performed a study on utilization of temporary ${ }^{125} \mathrm{I}$ in three groups: group A (38 cases) and B (40 cases) included patients with new brain metastases, and group C (21 cases) consisted of patients with recurrent brain metastases. In regards to location, 56 tumors were located in the cerebral hemispheres, 14 tumors were situated in the basal nuclei, 5 in the midbrain, 2 in the pons, and 6 tumors were located in the cerebellum. Primary tumors included 31 bronchial carcinomas, 21 hypernephromas, 18 melanomas, 18 GI tumors, 8 breast tumors, 3 uterine/ovarian tumors, two thyroid tumors, and two of unknown primaries. A radiation dose of $60 \mathrm{~Gy}$ was delivered at a dose rate of $7.2 \mathrm{cGy} / \mathrm{h}$. Group A was treated with brachytherapy with adjuvant RT, while groups $\mathrm{B}$ and $\mathrm{C}$ were treated with brachytherapy alone. At median follow-up of 3 months, they reported $100 \%$ of

Table 1. Isotopes used in studies evaluating brachytherapy in treatment of brain metastases

\begin{tabular}{|c|c|c|c|c|c|c|}
\hline Isotope & $\begin{array}{c}\text { Number of } \\
\text { studies }\end{array}$ & $\begin{array}{l}\text { Total \# of } \\
\text { patients of } \\
\text { studies }\end{array}$ & $\mathrm{mEV}$ & $\mathrm{t}_{1 / 2}$ (days) & $\begin{array}{l}\text { Half value } \\
\text { layer (mm } \\
\text { Pb) }\end{array}$ & Source \\
\hline $\begin{array}{l}125 \mid[8,15,17,18,19,20 \\
21,22,23,25,26,27,28 \\
29,30,31,32]\end{array}$ & 16 & 728 & $.0272-.0317$ & 59.4 days & 0.028 & $\begin{array}{l}\text { Neutron capture of } \\
{ }^{124} \mathrm{Xe} \rightarrow{ }^{125} \mathrm{Xe} \rightarrow{ }^{125} \mid \\
\text { (via electron capture) }\end{array}$ \\
\hline${ }^{131} \mathrm{Cs}[10,11,12,13,16]$ & 4 & $\begin{array}{c}79 \text { (two } \\
\text { studies used } \\
\text { same } 24 \text { pts) }\end{array}$ & $.0295-.0342$ & 9.7 days & & $\begin{array}{c}\text { Neutron activation of } \\
{ }^{130} \mathrm{Ba} \rightarrow{ }^{131} \mathrm{Ba} \rightarrow{ }^{131} \mathrm{Cs} \text { or } \\
\text { nuclear reaction of } \\
{ }^{133} \mathrm{Cs} \rightarrow{ }^{131} \mathrm{Ba} \rightarrow{ }^{131} \mathrm{Cs}\end{array}$ \\
\hline Photons $[8,9]$ & 2 & 78 & .01 to .02 & $10^{\wedge} 18$ yrs & 1 & $\begin{array}{l}\text { Delivery of electron beam } \\
\text { of } 40 \mu \mathrm{A} \text { through deflection } \\
\text { chamber, rigid probe, and } \\
\text { then thin gold foil }(0.5 \mu \mathrm{m}) \\
\text { producing photons with } \\
\text { energy } 10-20 \mathrm{kEv}\end{array}$ \\
\hline
\end{tabular}




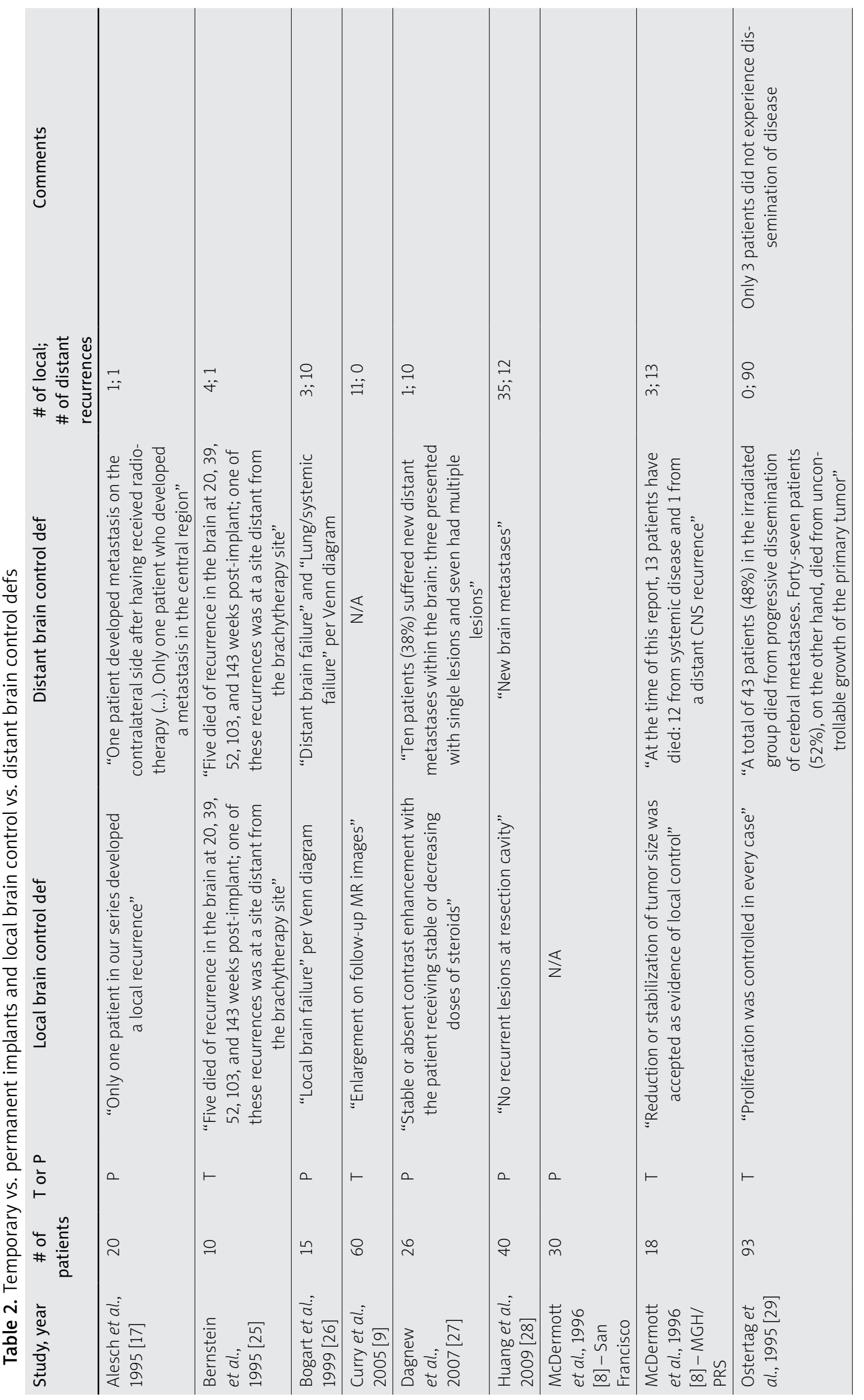




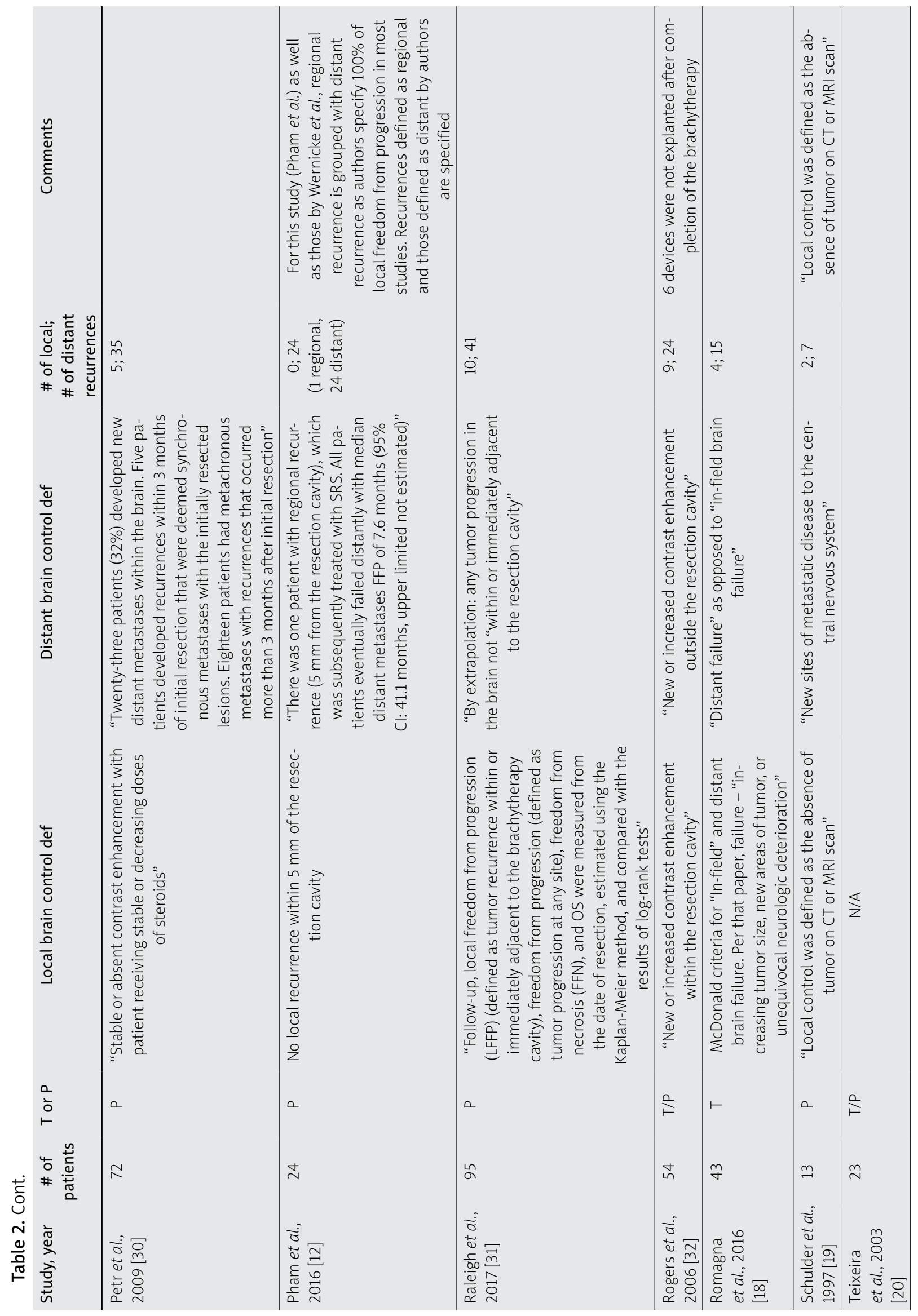




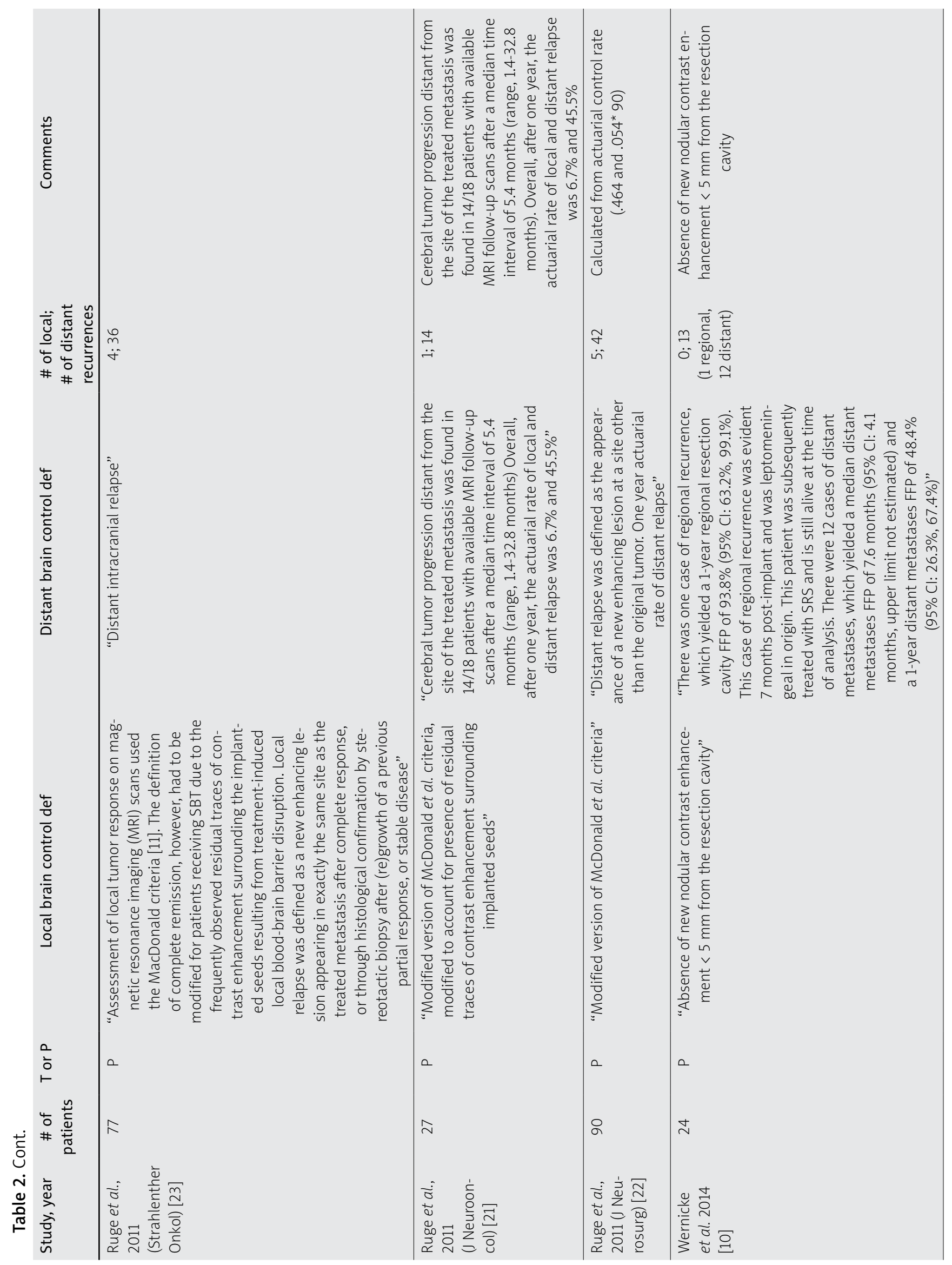




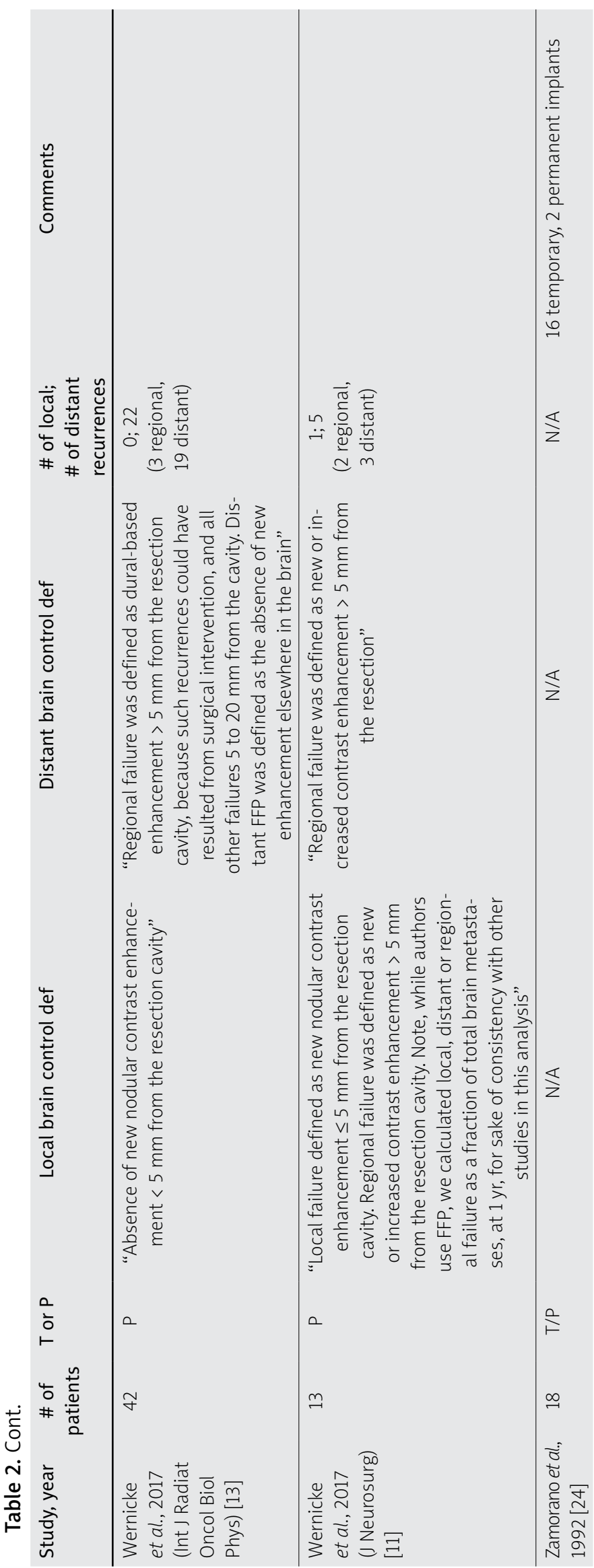

local control rate, however with $48 \%$ of distant recurrence (outside the resection cavity) rate (Table 4). The median overall survival was 17 months for group A, 15 months for group $B$, and 6 months for group C (Table 5). KPS was stable or improved in $79 \%$ of patients, and there were no cases of radiation necrosis. The only reported post-operative complication was transient hemiparesis in $2 \%$ of patients ( 2 patients in total) (Table 6 ). Their work showed that high rates of local control and KPS were possible with the use of the ${ }^{125} \mathrm{I}$ isotope for brachytherapy, even though the recurrence of disease at other brain sites remained a concern. Unfortunately, the prognosis of recurrent brain metastases was noticeably worse than that of new brain metastases, as indicated by significantly lower median OS in group C [29].

Petr et al. studied the use of surgical resection and permanent ${ }^{125} \mathrm{I}$ seeds for treatment of newly diagnosed brain single metastasis in 72 patients, between 1997 and 2007. Of the tumors treated, 66 were located in the cerebral hemispheres, 14 in the basal nuclei, 5 in the midbrain, 2 tumors were situated in the pons, and 6 in the cerebellum. Primary tumor sites included 38 lung (non-small cell lung cancer specifically), 9 breast, 6 colon, 5 melanoma, 3 ovarian, 3 renal, 1 prostate, 1 cervical, 1 bladder, and 4 of unknown malignancies (Table 3). A radiation dose of $150 \mathrm{~Gy}$ was delivered, with seed activity ranging from 4.04 to $40.38 \mathrm{mCi}$. They reported $93 \%$ of local control, distant brain failures in $32 \%$ of patients, and median OS of 14 months (Tables 4 and 5). The treatment was tolerable, and $100 \%$ of patients had stable or improved KPS. However, there was a $6 \%$ rate of radiation necrosis and $8 \%$ rate of other post-operative complications (Table 6). They demonstrated local control rates that compare favorably to WBRT while sparing patients' functional deterioration often associated with receiving WBRT, as indicated by stable or improved KPS in patients receiving brachytherapy. However, rates of distant recurrence were higher than in studies utilizing upfront WBRT [30].

Ruge and colleagues conducted a series of studies on ${ }^{125}$ I brachytherapy. The first of their studies compared permanent interstitial ${ }^{125} \mathrm{I}$ brachytherapy (77 patients) with stereotactic radiosurgery (142 patients) for treatment of de-novo singular brain metastases. Of these patients, 42 patients had disease in the cerebral hemispheres, 10 had tumors in the pons, 15 in the basal ganglia/diencephalon, 8 had disease in the cerebellum, and 2 had tumors located elsewhere. Primary sites included 20 lung tumors, 16 breast tumors, 3 melanomas, 3 colorectal tumors, 1 kidney tumor, 1 esophageal tumor, two tumors listed as other, and 1 of unknown primary (Table 3 ). Ruge et al. found that brachytherapy was overall comparable to SRS, with greater rates of local control vs. SRS, with $94.6 \%$ vs. $92.8 \%$, respectively, similar rates of distant control, with $53.6 \%$ vs. $57.6 \%$, respectively, and comparable median survival, with 8.0 vs. 8.1 months, respectively (Tables 4 and 5) [23]. The aim of their second study was to distinguish radiation-induced tumor changes and progression of disease in 30 patients with previously irradiated, locally recurrent brain metastases assessed with stereotactic biopsy. Twenty-seven of these patients had no 
Table 3. Tumor characteristics in studies evaluating brachytherapy in treatment of brain metastases

\begin{tabular}{|c|c|c|c|c|c|}
\hline Study, year & $\begin{array}{l}\text { \# of } \\
\text { patients }\end{array}$ & Primary tumor & Sites in brain & Implant & $\begin{array}{l}\text { Median } \\
\text { tumor } \\
\text { volume }\end{array}$ \\
\hline $\begin{array}{l}\text { Alesch et al., } \\
1995[17]\end{array}$ & 20 & $\begin{array}{l}\text { Lung (8), breast (3), colon (3), larynx (2), } \\
\text { kidney (1), thyroid (1) }\end{array}$ & $\begin{array}{l}\text { Frontal (8), parietal (5), temporal (3), } \\
\text { central (1), basal ganglia (2), pontine (1) }\end{array}$ & 125 & 4.2 \\
\hline $\begin{array}{l}\text { Bernstein } \\
\text { et al., } 1995 \\
{[25]}\end{array}$ & 10 & $\begin{array}{l}\text { Lung adenocarcinoma (9), breast ade- } \\
\text { nocarcinoma (1) }\end{array}$ & Cerebral hemispheres (9), cerebellar (1) & 125 & $36.4^{*}$ \\
\hline $\begin{array}{l}\text { Bogart } \\
\text { et al., } 1999 \\
{[26]}\end{array}$ & 15 & Lung (15; NSCLC) & $\begin{array}{l}\text { Frontal (5), parietal (5), occipital (4) } \\
\text { temporal (1) }\end{array}$ & 125 & 8.2 \\
\hline $\begin{array}{l}\text { Curry et al., } \\
2005 \text { [9] }\end{array}$ & 60 & $\begin{array}{l}\text { Lung (33), melanoma (15), renal cell } \\
\text { (5) breast (2), esophageal (2), colon (1), } \\
\text { and Merkle cell (1) malignant fibrous } \\
\text { histiocytoma (1) }\end{array}$ & $\begin{array}{l}\text { Frontal (29), frontoparietal (4), parietal } \\
\text { (13), temporal (17), temporoparietal (2), } \\
\text { parieto-occipital (1), occipital (4), basal } \\
\text { ganglia (1), cerebellar (1) }\end{array}$ & PRS & $7.8^{*}$ \\
\hline $\begin{array}{l}\text { Dagnew } \\
\text { et al., } 2007 \\
{[27]}\end{array}$ & 26 & \multicolumn{2}{|c|}{$\begin{array}{l}\text { Lung (12), melanoma (4) colon (3), breast (2), renal (1), cervix (1), prostate (1), } \\
\text { ovarian (1), unknown (1) }\end{array}$} & 125 & 14.1 \\
\hline $\begin{array}{l}\text { Huang et al. } \\
2009[28]\end{array}$ & 40 & $\begin{array}{l}\text { Melanoma (8), lung (7), breast (2), } \\
\text { other }(2)^{\star *}\end{array}$ & $\begin{array}{l}\text { Frontal (11), parietal (7), frontoparietal } \\
\text { (4), temporal (11), occipital (4), tem- } \\
\text { poro-occipital (1), occipitoparietal (1), } \\
\text { cerebellar (5) }\end{array}$ & 125 & 17.2 \\
\hline $\begin{array}{l}\text { McDermott } \\
\text { et al., } 1996 \\
\text { [8]-San } \\
\text { Francisco }\end{array}$ & 30 & $\begin{array}{l}\text { Adenocarcinoma (15), melanoma (8), } \\
\text { angiosarcoma (1), rhabdomyosarcoma } \\
\text { (1), Ewing's sarcoma, small cell carci- } \\
\text { noma (1), endometrial carcinoma (1), } \\
\text { undifferentiated sarcoma (1), } \\
\text { unknown (1) }\end{array}$ & N/A & 125 & $20.6^{\star}$ \\
\hline $\begin{array}{l}\text { McDermott } \\
\text { et al., } 1996 \\
\text { [8]-MGH/ } \\
\text { PRS }\end{array}$ & 18 & $\begin{array}{l}\text { Histology not specified; all lesions were } \\
\text { supratentorial }\end{array}$ & N/A & PRS & 4.9 \\
\hline $\begin{array}{l}\text { Ostertag } \\
\text { et al., } 1995 \\
{[29]}\end{array}$ & 93 & $\begin{array}{l}\text { Bronchial carcinoma (NSCLC; } 31) \text {, } \\
\text { hypernephroma (21), melanoma (18), } \\
\text { gastrointestinal (18), breast (8), uterus/ } \\
\text { ovary (3), thyroid (2), unknown (2) }\end{array}$ & $\begin{array}{l}\text { Cerebral hemispheres (66), basal nuclei } \\
\text { (14), midbrain (5), pons (2), cerebellar } \\
\text { (6) }\end{array}$ & 125 & 16.5 \\
\hline $\begin{array}{l}\text { Petr et al., } \\
2009[30]\end{array}$ & 72 & $\begin{array}{c}\text { Lung (38; NSCLC), breast (9), colon (6), } \\
\text { melanoma (5), ovarian (3), renal (3), } \\
\text { prostate (1), cervical (1), bladder (1), } \\
\text { unknown (4) }\end{array}$ & Supratentorial (55), infratentorial (17) & 125 & 14.1 \\
\hline $\begin{array}{l}\text { Pham et al., } \\
2016[12]\end{array}$ & 24 & $\begin{array}{l}\text { Lung (16), breast (2), kidney (2), melano- } \\
\text { ma (2), colon (1), cervix (1) }\end{array}$ & $\begin{array}{c}\text { Frontal (10), parietal (7), temporal (1), } \\
\text { occipital (2), cerebellar (4) }\end{array}$ & ${ }^{131} \mathrm{Cs}$ & 10.3 \\
\hline $\begin{array}{l}\text { Raleigh et } \\
\text { al., } 2017 \text { [31] }\end{array}$ & 95 & $\begin{array}{l}\text { Lung (36), melanoma (26), breast (22), } \\
\text { other (11) }\end{array}$ & $\begin{array}{l}\text { Frontal (32), parietal (17), temporal (26), } \\
\text { occipital (17), cerebellum (13), cerebral/ } \\
\text { cerebellar convexity (94), periventricular } \\
\text { (20), lobar tip (20) }\end{array}$ & 125 & 13.5 \\
\hline $\begin{array}{l}\text { Rogers } \\
\text { et al., } 2006 \\
{[32]}\end{array}$ & 54 & $\begin{array}{l}\text { Lung (29), gastrointestinal (7), melano- } \\
\text { ma (7), renal (3), other (8) }\end{array}$ & $\begin{array}{l}\text { Frontal (15), parietal (12), temporal (6), } \\
\text { occipital (7), other (14) }\end{array}$ & 125 & 14.1 \\
\hline $\begin{array}{l}\text { Romagna } \\
\text { et al., } 2016 \\
{[18]}\end{array}$ & 43 & $\begin{array}{c}\text { Lung (17; } 11 \text { NSCLC, } 2 \text { SCLC, } 4 \text { other), } \\
\text { skin (5), gastrointestinal (3),kidney (3), } \\
\text { uterus (1), ovary (1), musculoskeletal (1), } \\
\text { prostate (1) }\end{array}$ & N/A & 125 & 2.6 \\
\hline $\begin{array}{l}\text { Schulder } \\
\text { et al., } 1997 \\
\text { [19] }\end{array}$ & 13 & $\begin{array}{l}\text { Lung (4; NSCLC), breast (3), germ cell (3: } \\
\text { testicle 2, mediastinum 1), melanoma } \\
\text { (2), renal (1) }\end{array}$ & $\begin{array}{l}\text { Frontal (4), parietal (4), temporal (1), } \\
\text { occipital (1) }\end{array}$ & 125 & 14.1 \\
\hline
\end{tabular}


Table 3. Cont.

\begin{tabular}{|c|c|c|c|c|c|}
\hline Study, year & $\begin{array}{c}\text { \# of } \\
\text { patients }\end{array}$ & Primary tumor & Sites in brain & Implant & $\begin{array}{l}\text { Median } \\
\text { tumor } \\
\text { volume }\end{array}$ \\
\hline $\begin{array}{l}\text { Teixeira } \\
\text { et al., } 2003 \\
\text { [20] }\end{array}$ & 23 & $\begin{array}{c}\text { Lung (7), breast (4), other/unknown/ } \\
\text { undifferentiated (5) }\end{array}$ & $\begin{array}{c}\text { Including patients in study with primary } \\
\text { brain tumors (NOT just metastases) } \\
63 \% \text { of cases were in cerebral hemi- } \\
\text { spheres, } 21.8 \% \text { in deep structures, } 13.8 \% \\
\text { in brainstem }\end{array}$ & 125 & 38.3 \\
\hline $\begin{array}{l}\text { Ruge } \\
\text { et al., } 2011 \\
\text { (Strahlen- } \\
\text { ther Onkol) } \\
{[23]}\end{array}$ & 77 & $\begin{array}{c}\text { Lung (20; NSCLC), breast (16), kidney } \\
\text { (10), melanoma (7), colon (6), other (12), } \\
\text { unknown (6) }\end{array}$ & $\begin{array}{l}\text { Cerebral hemispheres (42), pons (10), } \\
\text { basal ganglia/diencephalon (15), cere- } \\
\text { bellar (8), other (2) }\end{array}$ & 125 & \\
\hline $\begin{array}{l}\text { Ruge et al., } \\
2011 \\
\text { (J Neuroon- } \\
\text { col) [21] }\end{array}$ & 27 & $\begin{array}{l}\text { Breast (11), lung (5; NSCLC) melanoma } \\
\text { (3), colorectal (3), kidney (1), esophagus } \\
\text { (1), other (2), unknown (1) }\end{array}$ & $\mathrm{N} / \mathrm{A}$ & 125 & \\
\hline $\begin{array}{l}\text { Ruge } \\
\text { et al., } 2011 \\
\text { (J Neurosurg) } \\
\text { [22] }\end{array}$ & 90 & $\begin{array}{l}\text { Lung (27; NSCLC), breast (17), kidney } \\
\text { (12), melanoma (8), colorectal (7), } \\
\text { other (13), unknown (6) }\end{array}$ & $\begin{array}{c}\text { Cerebral hemispheres (26), pons (12), } \\
\text { insular (6), pre/post central sulcus (19), } \\
\text { basal ganglia/diencephalon (13), } \\
\text { other (2) }\end{array}$ & 125 & * \\
\hline $\begin{array}{l}\text { Wernicke } \\
\text { et al., } 2014 \\
{[10]}\end{array}$ & 24 & $\begin{array}{l}\text { Lung (16), breast (2), kidney (2), melano- } \\
\text { ma (2), colon (1), cervix (1) }\end{array}$ & $\begin{array}{l}\text { Frontal (10), parietal (7), temporal (1), } \\
\text { occipital (2), cerebellar (4) }\end{array}$ & ${ }^{131} \mathrm{Cs}$ & 10.3 \\
\hline $\begin{array}{l}\text { Wernicke } \\
\text { et al., } 2017 \\
\text { (Int J Radiat } \\
\text { Oncol Biol } \\
\text { Phys) [13] }\end{array}$ & 42 & $\begin{array}{l}\text { Lung (26), colon (4), breast (3), melano- } \\
\text { ma (2), uterus ( } 2) \text {, esophagus (5), kidney } \\
\text { (1), hepatobiliary (1), tonsillar (1) }\end{array}$ & $\begin{array}{l}\text { Frontal (14), parietal (14), temporal (4), } \\
\text { occipital (3), cerebellar (11) }\end{array}$ & ${ }^{131} \mathrm{Cs}$ & 14.1 \\
\hline $\begin{array}{l}\text { Wernicke } \\
\text { et al., } 2017 \\
\text { (J Neuro- } \\
\text { surg) [11] }\end{array}$ & 13 & $\begin{array}{l}\text { Lung (9), melanoma (3), breast (1), } \\
\text { gastric (1), pancreatic (1) }\end{array}$ & $\begin{array}{l}\text { Frontal (3), parietal (4), temporal (3), } \\
\text { occipital (2), cerebellar (2), insular (1) }\end{array}$ & ${ }^{131} \mathrm{Cs}$ & 12.8 \\
\hline $\begin{array}{l}\text { Zamorano } \\
\text { et al., } 1992 \\
{[24]}\end{array}$ & 18 & N/A & N/A & 125 & \\
\hline
\end{tabular}

*Most volumes listed were calculated from tumor diameter via $4 / 3$ w $(D / 2)^{3}$ and represent median volume.

Exceptions: Bernstein et al., 1995 [25]: volume listed is implant volume, Curry et al., 2005 [9]: volume listed is mean treatment volume, Ruge et al., 2011 [22] (J Neurosurg): 70 patients had tumor volume $<14 \mathrm{~cm}, 20$ patients had tumor volume > 14 cm; McDermott et al., 1996 [8] San Francisco: volume listed = isodose volume

signs of radiation necrosis on biopsy, and received 50 Gy of permanent ${ }^{125} \mathrm{I}$ brachytherapy for 42 days (Table 6). Primary tumors among treated patients included 11 breast, 5 lung (non-small cell lung cancer), 3 melanoma, 3 colorectal, 1 kidney, 1 esophagus, two other, and one of unknown origin (Table 3). Their rates of local and distant control were $92.3 \%$ and $54.5 \%$, respectively, with median overall survival of 14.8 months (Tables 4 and 5). Furthermore, $94 \%$ of patients displayed stable or improved KPS at 3 months follow-up. No patients experienced radionecrosis, and $6.6 \%$ of patients experienced post-operative complications, including one with a wound infection and one with transient aphasia (Table 6) [21]. Their third study included 90 patients with singular brain metastases treated with stereotactic permanent ${ }^{125} \mathrm{I}$ brachytherapy. Of these, 26 patients had primary tumors of the lung, 17 of the breast, 12 of the kidney, 8 melanomas, 7 colorec- tal tumors, 13 tumors of other primary site, and 6 tumors of unknown primary site. Locations of these tumors included 26 tumors in the cerebral hemispheres, 12 tumors in the pons, 6 insular tumors, 19 pre/post-central sulcus, 13 basal ganglia/diencephalon, and 2 in another locations (Table 3). They found that brachytherapy compared well to other local therapies, namely surgery and SRS, with rates of local disease control of $94.6 \%$, distant disease control of $53.6 \%$, and median overall survival of 8.5 months (Tables 4 and 5). Of note, only $4.4 \%$ of patients experienced post-operative complications, including acute renal failure post-surgery (1 case), superficial wound infection (2 cases), and CSF fistula (1 case) (Table 6) [22].

These large studies evaluating ${ }^{125} \mathrm{I}$ brachytherapy demonstrate that excellent rates of local control, good rates of overall survival, and improvements in quality of life were possible to achieve. However, rates of regional 
Table 4. Extent of local brain control in studies evaluating brachytherapy in treatment of brain metastases

\begin{tabular}{|c|c|c|c|c|c|}
\hline Study, year & $\begin{array}{c}\text { \# of } \\
\text { patients }\end{array}$ & Implant & $\begin{array}{l}\text { Fxn with local } \\
\text { brain control }\end{array}$ & $\begin{array}{l}\text { Time used } \\
\text { for LBC/FFP }\end{array}$ & LBC def \\
\hline $\begin{array}{l}\text { Alesch et al., } 1995 \\
\text { [17] }\end{array}$ & 20 & 125 & $95 \%$ & & No local progression \\
\hline $\begin{array}{l}\text { Bernstein et al., } 1995 \\
{[25]}\end{array}$ & 10 & 125 & $40 \%$ & 81 & No local recurrence \\
\hline $\begin{array}{l}\text { Bogart et al., } 1999 \\
{[26]}\end{array}$ & 15 & 125 & $66 \%$ & & No recurrent at or adjacent to primary site \\
\hline Curry et al., 2005 [9] & 60 & PRS & $81 \%$ & 6 & $\begin{array}{c}\text { Demonstrated stabilization or reduction in tumor } \\
\text { size on MRI }\end{array}$ \\
\hline $\begin{array}{l}\text { Dagnew et al., } 2007 \\
\text { [27] }\end{array}$ & 26 & 125 & $96 \%$ & 12 & $\begin{array}{l}\text { Stable or absent contrast enhancement with patient } \\
\text { receiving stable or decreasing doses of steroids }\end{array}$ \\
\hline $\begin{array}{l}\text { Huang et al., } 2009 \\
\text { [28] }\end{array}$ & 40 & 125 & $88 \%$ & 12 & No recurrent lesions at resection cavity \\
\hline $\begin{array}{l}\text { McDermott et al., } \\
1996[8] \text { - San Fran- } \\
\text { cisco }\end{array}$ & 30 & 125 & & $14.5-49$ & N/A \\
\hline $\begin{array}{l}\text { McDermott et al., } \\
1996[8]-\text { MGH/PRS }\end{array}$ & 18 & PRS & $83 \%$ & $1.5-24$ & $\begin{array}{l}\text { Reduction or stabilization of tumor size was accept- } \\
\text { ed as evidence of local control }\end{array}$ \\
\hline $\begin{array}{l}\text { Ostertag et al., } 1995 \\
\text { [29] }\end{array}$ & 93 & 125 & $100 \%$ & 3 & Proliferation was controlled in every case \\
\hline Petr et al., 2009 [30] & 72 & 125 & $93 \%$ & & $\begin{array}{l}\text { Stable or absent contrast enhancement with patient } \\
\text { receiving stable or decreasing doses of steroids }\end{array}$ \\
\hline Pham et al., 2016 [12] & 24 & ${ }^{131} \mathrm{Cs}$ & $100 \%$ & 19.3 & $\begin{array}{l}\text { No local recurrence within } 5 \mathrm{~mm} \text { of the resection } \\
\text { cavity }\end{array}$ \\
\hline $\begin{array}{l}\text { Raleigh et al., } 2017 \\
\text { [31] }\end{array}$ & 95 & 125 & $90 \%$ & 14.4 & $\begin{array}{l}\text { Local freedom from progression (i.e. no tumor } \\
\text { recurrence within or immediately adjacent to the } \\
\text { brachytherapy cavity) }\end{array}$ \\
\hline $\begin{array}{l}\text { Rogers et al., } 2006 \\
\text { [32] }\end{array}$ & 54 & 125 & $83 \%$ & 12 & $\begin{array}{l}\text { New or increased contrast enhancement within the } \\
\text { resection cavity }\end{array}$ \\
\hline $\begin{array}{l}\text { Romagna et al., } 2016 \\
{[18]}\end{array}$ & 43 & 125 & $91 \%$ & 12 & $\begin{array}{c}\text { McDonald criteria for "in-field" and distant brain } \\
\text { failure. Per that paper, failure = "increasing tumor } \\
\text { size, new areas of tumor, or unequivocal neurologic } \\
\text { deterioration" }\end{array}$ \\
\hline $\begin{array}{l}\text { Schulder et al., } 1997 \\
\text { [19] }\end{array}$ & 13 & 125 & $69 \%$ & & $\begin{array}{l}\text { Local control was defined as the absence of tumor } \\
\text { on CT or MRI scan }\end{array}$ \\
\hline $\begin{array}{l}\text { Teixeira et al., } 2003 \\
\text { [20] }\end{array}$ & 23 & 125 & & & $\mathrm{~N} / \mathrm{A}$ \\
\hline $\begin{array}{l}\text { Ruge et al., } 2011 \\
\text { (Strahlenther Onkol) } \\
\text { [23] }\end{array}$ & 77 & 125 & $95 \%$ & 12 & $\begin{array}{l}\text { Assessment of local tumor response on magnetic } \\
\text { resonance imaging (MRI) scans used the MacDonald } \\
\text { criteria [11]. The definition of complete remission, } \\
\text { however, had to be modified for patients receiving } \\
\text { SBT due to the frequently observed residual traces } \\
\text { of contrast enhancement surrounding the implanted } \\
\text { seeds resulting from treatment-induced local blood- } \\
\text { brain barrier disruption. Local relapse was defined } \\
\text { as a new enhancing lesion appearing in exactly the } \\
\text { same site as the treated metastasis after complete } \\
\text { response, or through histological confirmation by } \\
\text { stereotactic biopsy after (re)growth of a previous par- } \\
\text { tial response, or stable disease }\end{array}$ \\
\hline $\begin{array}{l}\text { Ruge et al., } 2011 \\
\text { (J Neurooncol) [21] }\end{array}$ & 27 & 125 & $92 \%$ & 12 & $\begin{array}{l}\text { Modified version of McDonald et al. criteria, mod- } \\
\text { ified to account for presence of residual traces of } \\
\text { contrast enhancement surrounding implanted seeds }\end{array}$ \\
\hline
\end{tabular}


Table 4. Cont.

\begin{tabular}{|c|c|c|c|c|c|}
\hline Study, year & $\begin{array}{c}\text { \# of } \\
\text { patients }\end{array}$ & Implant & $\begin{array}{l}\text { Fxn with local } \\
\text { brain control }\end{array}$ & $\begin{array}{l}\text { Time used } \\
\text { for LBC/FFP }\end{array}$ & LBC def \\
\hline $\begin{array}{l}\text { Ruge et al., } 2011 \\
\text { (J Neurosurg) [22] }\end{array}$ & 90 & 125 & $98 \%$ & 12 & Modified version of McDonald et al. criteria \\
\hline $\begin{array}{l}\text { Wernicke et al., } 2014 \\
{[10]}\end{array}$ & 24 & ${ }^{131} \mathrm{Cs}$ & $100 \%$ & 12 & $\begin{array}{l}\text { Absence of new nodular contrast enhancement } \\
\qquad 55 \mathrm{~mm} \text { from the resection cavity }\end{array}$ \\
\hline $\begin{array}{l}\text { Wernicke et al., } 2017 \\
\text { (Int J Radiat Oncol } \\
\text { Biol Phys) [13] }\end{array}$ & 42 & ${ }^{131} \mathrm{Cs}$ & $100 \%$ & 12 & $\begin{array}{l}\text { Absence of new nodular contrast enhancement } \\
\qquad<5 \mathrm{~mm} \text { from the resection cavity }\end{array}$ \\
\hline $\begin{array}{l}\text { Wernicke et al., } 2017 \\
\text { (J Neurosurg) [11] }\end{array}$ & 13 & ${ }^{131} \mathrm{Cs}$ & $93 \%$ & 12 & $\begin{array}{l}\text { Local failure defined as new nodular contrast } \\
\text { enhancement } \leq 5 \mathrm{~mm} \text { from the resection cavity. } \\
\text { Regional failure was defined as new or increased } \\
\text { contrast enhancement }>5 \mathrm{~mm} \text { from the resection } \\
\text { cavity. Note, while authors use FFP, we calculated } \\
\text { local, distant or regional failure as a fraction of total } \\
\text { brain metastases, at } 1 \mathrm{yr} \text {, for sake of consistency with } \\
\text { other studies in this analysis }\end{array}$ \\
\hline $\begin{array}{l}\text { Zamorano et al., } 1992 \\
\text { [24] }\end{array}$ & 18 & 125 & $\mathrm{~N} / \mathrm{A}$ & N/A & N/A \\
\hline
\end{tabular}

recurrence, rates of radiation necrosis, and other post-operative complications needed an improvement.

\section{Photon radiosurgery}

In addition to ${ }^{125} \mathrm{I}$ brachytherapy, some studies have examined the use of photon radiosurgery (PRS) as a modality of brachytherapy for brain metastases $[8,9]$. The photon radiosurgery device (Photoelectron Corp, Lexington, MA, United States) consist of a miniaturized X-ray source at the end of a small minimally invasive interstitial probe. Electrons from a small battery-powered thermionic gun are accelerated to a final energy of up to $40 \mathrm{keV}$ and directed along a tube to a thin Au target, where the beam size is approximately $0.3 \mathrm{~mm}$. X-ray output, which is nearly isotropic, consists of a bremsstrahlung spectrum and several lines between 7 and $14 \mathrm{keV}$ [33]. In a study of McDermott et al., PRS doses ranging from 10-26 Gy were used with WBRT for treatment of 18 patients with supratentorial brain metastases (Table 3). Local control rates of $83 \%$ was achieved, with regional recurrence in only 1 of 18 patients $(5.6 \%)$ and transient acute post-op complications in $22 \%$ of patients (Tables 4 and 6 ). Additionally, a greater control of radioresistant lesions with PRS was obtained compared to $90 \%$ of external radiosurgery [8]. Curry et al. delivered stereotactic low activity photons via a photon radiosurgery system (PRS) for treatment of 60 brain metastases. Tumor locations included frontal lobe (29 of patients), frontoparietal (4), parietal (13), temporal (17), temporoparietal (2), parieto-occipital (1), occipital (4), basal ganglia (1), and cerebellar (1 case). Primary tumor sites included 33 lung tumors, 15 melanoma, 5 renal, 2 breast, 2 esophagus, 1 colon, 1 Merkle cell, and 1 malignant fibrous astrocytoma (Table 3 ). Local brain control rate of $81.4 \%$ was achieved, with median OS of 8 months (Table 4 and 5). There was a radiation necrosis rate of $5 \%$ and a $15 \%$ rate of other acute post-operative complications (Table 6) [9].

\section{Cesium-131}

Most studies on ${ }^{131} \mathrm{Cs}$ brachytherapy for treatment of brain metastases have been performed by Wernicke and colleagues including 24 patients in two studies and 42 in another research. Patients were treated with local resection, followed by implantation of permanent ${ }^{131} \mathrm{Cs}$ seeds (Table 2) $[10,11,12,13]$. These studies reported $100 \%$ of local brain control, low rates of regional recurrence, and distant progression within the brain, with no cases of radiation necrosis and minimal post-operative complications (Tables 4 and 6). Their first study involved 24 patients, with disease sites including 10 frontal, 7 parietal, 4 cerebellar, 2 occipital, and 1 temporal tumor. Primary tumors consisted of 16 lung, 2 breast, 2 kidney, 2 melanoma, 1 colon, and 1 cervix cancer. They delivered an 80 Gy dose at $5 \mathrm{~mm}$ depth from the resection cavity. With median follow-up of 12 months, they achieved $100 \%$ rate of local control, with regional recurrence rate of $6.2 \%$, distant recurrence rate of $51.6 \%$, and median OS of 9.9 months (Table 5). There were no cases of radiation necrosis, although complications occurred in $12.5 \%$ of patients and included a cerebrospinal fluid leak, a seizure, and an infection (Table 6) [10].

Their second study assessed the use of ${ }^{131} \mathrm{Cs}$ brachytherapy for large tumors, defined as tumors $>2.0 \mathrm{~cm}$ in diameter, which historically have higher rate of radiation necrosis as well as recurrence. Stereotactic radiosurgery (SRS), which generally offers excellent local control suffers from high rates of recurrence in large tumors $>3.0 \mathrm{~cm}$ in diameter. In a phase 2 trial of SRS by Brennan et al., a 2-year actuarial control rate was achieved in only $40 \%$ in tumors $>3.0 \mathrm{~cm}$ vs. $89 \%$ in those $<3.0 \mathrm{~cm}[34,35]$. A study done by Wernicke et al. included 42 patients, with 14 parietal, 14 frontal,11 cerebellar, 3 occipital, and 4 temporal metastases. Histology featured 26 lung, 4 colon, 3 breast, 2 melanoma, 2 uterine, 2 esophageal, 1 kidney, 1 hepatobiliary, and 1 tonsillar tumor (Table 3 ). Their 
Table 5. Survival rates in studies evaluating brachytherapy in treatment of brain metastases

\begin{tabular}{|c|c|c|c|c|}
\hline Study, year & \# of patients & Implant & 12 months survival rate & $\begin{array}{l}\text { Median overall survival } \\
\text { (months) }\end{array}$ \\
\hline Alesch et al., 1995 [17] & 20 & 125 & & \\
\hline Bernstein et al., 1995 [25] & 10 & 125 & $50 \%$ & 11.5 \\
\hline Bogart et al., 1999 [26] & 15 & 125 & $13 \%$ & 14 \\
\hline Curry et al., 2005 [9] & 60 & PRS & $34 \%$ & 8 \\
\hline Dagnew et al., 2007 [27] & 26 & 125 & $72 \%$ & 17.8 \\
\hline Huang et al., 2009 [28] & 40 & 125 & $48 \%$ & 11.3 \\
\hline $\begin{array}{l}\text { McDermott et al., } 1996 \text { [8] - } \\
\text { San Francisco }\end{array}$ & 30 & 125 & $55 \%$ & 14.7 \\
\hline $\begin{array}{l}\text { McDermott et al., } 1996 \text { [8] - } \\
\text { MGH/PRS }\end{array}$ & 18 & PRS & & \\
\hline Ostertag et al., 1995 [29] & 93 & 125 & $\begin{array}{c}\text { Lung - } 42 \% \text {, hypernephroma - } \\
66 \% \text {, melanoma }-50 \%\end{array}$ & $\begin{array}{c}17 \text { (group A), } 15 \text { (group B), } \\
6 \text { (group C) }\end{array}$ \\
\hline Petr et al., 2009 [30] & 72 & 125 & $55 \%$ & 14 \\
\hline Pham et al., 2016 [12] & 24 & ${ }^{131} \mathrm{Cs}$ & & \\
\hline Raleigh et al., 2017 [31] & 95 & 125 & & 12 \\
\hline Rogers et al., 2006 [32] & 54 & 125 & $40 \%$ & 40 \\
\hline Romagna et al., 2016 [18] & 43 & 125 & & 21.2 \\
\hline Schulder et al., 1997 [19] & 13 & 125 & $38 \%$ & 9 \\
\hline Teixeira et al., 2003 [20] & 23 & 125 & $\geq 40 \%$ & 10 \\
\hline $\begin{array}{l}\text { Ruge et al., } 2011 \text { (Strahlenther } \\
\text { Onkol) [23] }\end{array}$ & 77 & 125 & & 8 \\
\hline $\begin{array}{l}\text { Ruge et al., } 2011 \text { (J Neurooncol) } \\
\text { [21] }\end{array}$ & 27 & 125 & & 14.8 \\
\hline $\begin{array}{l}\text { Ruge et al., } 2011 \text { (J Neurosurg) } \\
\text { [22] }\end{array}$ & 90 & 125 & & 8.5 \\
\hline Wernicke et al., 2014 [10] & 24 & ${ }^{131} \mathrm{Cs}$ & $50 \%$ & 9.9 \\
\hline $\begin{array}{l}\text { Wernicke et al., } 2017 \text { (Int J Ra- } \\
\text { diat Oncol Biol Phys) [13] }\end{array}$ & 42 & ${ }^{131} \mathrm{Cs}$ & $58 \%$ & 15.1 \\
\hline $\begin{array}{l}\text { Wernicke et al., } 2017 \text { (J Neuro- } \\
\text { surg) [11] }\end{array}$ & 13 & ${ }^{131} \mathrm{Cs}$ & $25 \%$ & 7 \\
\hline Zamorano et al., 1992 [24] & 18 & 125 & $44 \%$ & 11 \\
\hline
\end{tabular}

disease control rates included $100 \%$ of local control rate, additionally noted a $7.1 \%$ of regional recurrence rate, distant recurrence rate of $54 \%$ at 12 months, and overall survival of 15.1 months (Tables 4 and 5). While no case of radiation necrosis was reported, complications were seen in $26 \%$ of patients, including 6 seizures in patients with no prior history of seizures, one intracranial infection, one case of brachytherapy seed migration, and superficial wound infections seen in 3 patients, one of whom also had a CSF leak (Table 6).

In addition to the aforementioned studies, Wernicke et al. conducted a research utilizing ${ }^{131} \mathrm{C}$ s brachytherapy as a salvage treatment, including 13 patients with recurrent brain metastases resistant to SRS and/or WBRT. Of these, 3 tumors were in the frontal lobe, 4 parietal, 2 occipital,
3 temporal, 2 cerebellar, and 1 insular. Histology included 9 lung tumors, 3 melanomas, 1 breast, 1 pancreatic, and 1 gastric tumor (Table 3 ). The prescription dose was 80 Gy located at $5 \mathrm{~mm}$ from the resection cavity surface. The 1-year local control rate was $93.3 \%$, with $13.3 \%$ of regional recurrence and $20 \%$ of distant recurrence (Table 4 ). In a median OS of 7 months, radiation necrosis rate was $0 \%$; however, a rate of acute post-operative complications occurred in $46 \%$ of patients (Tables 5 and 6). This was attributed to poor general condition of patients and small size of investigated cohort [11].

Studies on standard of care therapies for brain metastases, e.g. WBRT and SRS, have demonstrated that the treatment with these modalities may lead to an acute decline in cognitive function, as measured by FACT-BR 
Table 6. Treatment complications in studies evaluating brachytherapy in treatment of brain metastases

\begin{tabular}{|c|c|c|c|c|c|c|c|}
\hline Study, year & $\begin{array}{c}\text { \# of } \\
\text { patients }\end{array}$ & Implant & Necrosis & $\begin{array}{l}\text { Fxn other } \\
\text { acute post- } \\
\text { op compli- } \\
\text { cation }\end{array}$ & $\begin{array}{l}\text { Comments on acute } \\
\text { post-op complication }\end{array}$ & $\begin{array}{l}\text { Fxn with other } \\
\text { complication } \\
\text { caused by } \\
\text { implant }\end{array}$ & $\begin{array}{l}\text { Comment on } \\
\text { other compli- } \\
\text { cation }\end{array}$ \\
\hline $\begin{array}{l}\text { Alesch et al., } 1995 \\
\text { [17] }\end{array}$ & 20 & 125 & $0 \%$ & $0 \%$ & $N / A$ & $0 \%$ & N/A \\
\hline $\begin{array}{l}\text { Bernstein et al., } \\
1995 \text { [25] }\end{array}$ & 10 & 125 & $30 \%$ & $20 \%$ & $\begin{array}{l}\text { Both had suspected } \\
\text { pulmonary embolus }\end{array}$ & $20 \%$ & $\begin{array}{c}\text { Both had } \\
\text { permanent } \\
\text { worsening of } \\
\text { pre-existing } \\
\text { motor weak- } \\
\text { ness }\end{array}$ \\
\hline $\begin{array}{l}\text { Bogart et al., } 1999 \\
\text { [26] }\end{array}$ & 15 & 125 & $0 \%$ & $7 \%$ & 1 fungal infection & $0 \%$ & N/A \\
\hline $\begin{array}{l}\text { Curry et al., } 2005 \\
\text { [9] }\end{array}$ & 60 & PRS & $5 \%$ & $15 \%$ & $\begin{array}{l}\text { Post-op seizures (4), } \\
\text { cerebral edema (3), } \\
\text { hemorrhage ( } 2 \text { ), also } \\
\text { not included - radia- } \\
\text { tion necrosis = } 3\end{array}$ & N/A & N/A \\
\hline $\begin{array}{l}\text { Dagnew et al., } \\
2007 \text { [27] }\end{array}$ & 26 & 125 & $3 \%$ & N/A & N/A & N/A & N/A \\
\hline $\begin{array}{l}\text { Huang et al., } 2009 \\
\text { [28] }\end{array}$ & 40 & 125 & $23 \%$ & N/A & N/A & $2.5 \%$ & $\begin{array}{l}1 \text { patient had } \\
\text { mild perma- } \\
\text { nent progres- } \\
\text { sive speech } \\
\text { hesitancy }\end{array}$ \\
\hline $\begin{array}{l}\text { McDermott et al., } \\
1996[8]-\text { San } \\
\text { Francisco }\end{array}$ & 30 & 125 & $10 \%$ & N/A & N/A & N/A & N/A \\
\hline $\begin{array}{l}\text { McDermott et al., } \\
1996 \text { [8]-MGH/ } \\
\text { PRS }\end{array}$ & 18 & PRS & N/A & $22 \%$ & $\begin{array}{l}\text { Transient new neu- } \\
\text { rologic deficits (2), } \\
\text { partial seizures (2) }\end{array}$ & $0 \%$ & N/A \\
\hline $\begin{array}{l}\text { Ostertag et al., } \\
1995 \text { [29] }\end{array}$ & 93 & $125 \mid$ & $0 \%$ & $2 \%$ & $\begin{array}{c}\text { Transient hemiparesis } \\
\text { (2) }\end{array}$ & N/A & N/A \\
\hline $\begin{array}{l}\text { Petr et al., } 2009 \\
\text { [30] }\end{array}$ & 72 & 125 & $6 \%$ & $8 \%$ & $\begin{array}{l}7 \% \text { had thromboem- } \\
\text { bolic events, } 1 \% \text { had } \\
\text { a post-op infection }\end{array}$ & N/A & N/A \\
\hline $\begin{array}{l}\text { Pham et al., } 2016 \\
\text { [12] }\end{array}$ & 24 & ${ }^{131} \mathrm{Cs}$ & $0 \%$ & N/A & N/A & N/A & N/A \\
\hline $\begin{array}{l}\text { Raleigh et al., } \\
2017 \text { [31] }\end{array}$ & 95 & 125 & $15 \%$ & $6 \%$ & Wound complication & N/A & N/A \\
\hline $\begin{array}{l}\text { Rogers et al., } \\
2006[32]\end{array}$ & 54 & 125 & $7 \%$ & $13 \%$ & $\begin{array}{l}1 \text { each of grade } 3 \text { CSF } \\
\text { leak, headache, hemi- } \\
\text { plegia, hydrocephalus, } \\
\text { infection, intracranial } \\
\text { hemorrhage and } \\
\text { grade } 2 \text { seizure }\end{array}$ & N/A & N/A \\
\hline $\begin{array}{l}\text { Romagna et al., } \\
2016 \text { [18] }\end{array}$ & 43 & $125 \mid$ & $0 \%$ & N/A & N/A & N/A & N/A \\
\hline $\begin{array}{l}\text { Schulder et al., } \\
1997 \text { [19] }\end{array}$ & 13 & 125 & $15 \%$ & $15 \%$ & $\begin{array}{l}\text { Intracerebral hema- } \\
\text { toma/PE in one, and } \\
\text { ARDS in another }\end{array}$ & $\begin{array}{l}\text { 15\% (1 bone flap } \\
\text { infection, } 1 \text { CSF } \\
\text { leak, both treat- } \\
\text { ed w/o further } \\
\text { sequalae) }\end{array}$ & N/A \\
\hline
\end{tabular}


Table 6. Cont.

\begin{tabular}{|c|c|c|c|c|c|c|c|}
\hline Study, year & $\begin{array}{c}\text { \# of } \\
\text { patients }\end{array}$ & Implant & Necrosis & $\begin{array}{l}\text { Fxn other } \\
\text { acute post- } \\
\text { op compli- } \\
\text { cation }\end{array}$ & $\begin{array}{l}\text { Comments on acute } \\
\text { post-op complication }\end{array}$ & $\begin{array}{l}\text { Fxn with other } \\
\text { complication } \\
\text { caused by } \\
\text { implant }\end{array}$ & $\begin{array}{l}\text { Comment on } \\
\text { other compli- } \\
\text { cation }\end{array}$ \\
\hline $\begin{array}{l}\text { Teixeira et al., } \\
2003[20]\end{array}$ & 23 & 125 & N/A & $5 \%$ & $\begin{array}{l}\text { 7/138; } 5 \text { patients had } \\
\text { infection - } 3 \text { with skin } \\
\text { infection and } 2 \text { with } \\
\text { osteomyelitis and } \\
2 \text { patients had inci- } \\
\text { sional CSF leakage }\end{array}$ & $\mathrm{N} / \mathrm{A}$ & N/A \\
\hline $\begin{array}{l}\text { Ruge et al., } 2011 \\
\text { (Strahlenther } \\
\text { Onkol) [23] }\end{array}$ & 77 & 125 & $0 \%$ & $\mathrm{~N} / \mathrm{A}$ & N/A & N/A & N/A \\
\hline $\begin{array}{l}\text { Ruge et al., } 2011 \\
\text { (J Neurooncol) [21] }\end{array}$ & 27 & 125 & $0 \%$ & $7 \%$ & $\begin{array}{l}1 \text { patient developed } \\
\text { wound infection, } 1 \\
\text { patient developed } \\
\text { transient aphasia }\end{array}$ & $\mathrm{N} / \mathrm{A}$ & N/A \\
\hline $\begin{array}{l}\text { Ruge et al., } 2011 \\
\text { (J Neurosurg) [22] }\end{array}$ & 90 & 125 & & $4 \%$ & $\begin{array}{l}\text { Acute renal failure } \\
\text { post-surgery (1), } \\
\text { superficial wound } \\
\text { infection (2), CSF } \\
\text { fistula (1) }\end{array}$ & N/A & N/A \\
\hline $\begin{array}{l}\text { Wernicke et al., } \\
2014 \text { [10] }\end{array}$ & 24 & ${ }^{131} \mathrm{Cs}$ & $0 \%$ & $13 \%$ & $\begin{array}{l}\text { CSF leak (1), seizure } \\
\text { (1), infection (1) }\end{array}$ & $\mathrm{N} / \mathrm{A}$ & N/A \\
\hline $\begin{array}{l}\text { Wernicke et al., } \\
2017 \text { (Int J Radiat } \\
\text { Oncol Biol Phys) } \\
\text { [13] }\end{array}$ & 42 & ${ }^{131} \mathrm{Cs}$ & $0 \%$ & $26 \%$ & $\begin{array}{l}11 \text { - seizures (6, in } \\
\text { patients w/no hx of } \\
\text { seizures), superficial } \\
\text { wound infections (3), } \\
\text { CSF leak (1 patient } \\
\text { who already de- } \\
\text { veloped superficial } \\
\text { wound infection), } \\
\text { intracranial infection } \\
\text { (1), } 1 \text { who developed } \\
\text { brachytherapy seed } \\
\text { migration }\end{array}$ & N/A & N/A \\
\hline $\begin{array}{l}\text { Wernicke et al., } \\
2017 \text { (J Neuro- } \\
\text { surg) [11] }\end{array}$ & 13 & ${ }^{131} \mathrm{Cs}$ & $0 \%$ & $46 \%$ & $\begin{array}{c}3 \text { infections, } 1 \text { seizures } \\
\text { and } 1 \text { pseudo-menin- } \\
\text { gocele }\end{array}$ & $N / A$ & N/A \\
\hline $\begin{array}{l}\text { Zamorano et al., } \\
1992[24]\end{array}$ & 18 & 125 & N/A & N/A & Worsened KPS after tx & $\begin{array}{c}33 \%(5 / 16 \\
\text { temporary, and } \\
1 / 2 \text { permanent } \\
\text { implants) }\end{array}$ & $\begin{array}{c}\text { Remaining } \\
67 \%(11 / 16 \\
\text { temporary and } \\
1 / 2 \text { permanent) } \\
\text { had stable or } \\
\text { improved KPS }\end{array}$ \\
\hline
\end{tabular}

questionnaire [36,37]. This questionnaire assesses physical, functional, and emotional well-being. Irrespective of treatment modality, radiologic control of disease was associated with decreased decline in cognitive function, as measured by the mini-mental status exam (MMSE) score [38]. A decline in scores over 3 months was 0.5 for those with well controlled disease vs. that of poorly radiologically controlled, with a decline of 6.3. The first evaluation of ${ }^{131} \mathrm{Cs}$ brachytherapy per these indices showed a promise. Pham et al. found that ${ }^{131} \mathrm{Cs}$ brachytherapy at least preserves quality of life in patients with brain metasta- ses, on the basis of FACT-BR questionnaire score increase from 146.5 to 164 at 6 months post-treatment. Furthermore, an improvement in MMSE score of all patients was observed, including patients with a pretreatment MMSE score $<27$ with an increase to a score of 30 [12].

\section{Discussion}

The purpose of this review was to provide a summary of the published data using brachytherapy for the treatment of brain metastases. Goals included identifying 
brachytherapy techniques with the most supportive data, and recognizing important questions to improve the efficacy and safety of this treatment modality.

The majority of data on treatment of brain metastases with brachytherapy uses the ${ }^{125} \mathrm{I}$ isotope. ${ }^{125} \mathrm{I}$ brachytherapy produces excellent rates of local control and overall survival as well as improvements in KPS score [21,29,30]. It additionally demonstrates a promise as an effective salvage therapy for recurrent brain metastases [21]. Unfortunately, this technique tends to result in high rates of radiation necrosis, and post-operative complications may explain why brachytherapy has not been commonly used in the treatment of brain metastases [21,30]. This is particularly important because not only can radiation necrosis be symptomatic, but even when asymptomatic, it may preclude further therapy [21]. Due to the heterogeneity of the studies and different reporting methods, conclusions regarding the rates of symptomatic versus asymptomatic radiation necrosis were not established.

As an alternate method of brachytherapy, the photon radiosurgery (form of electronic brachytherapy) device has been presented. PRS is limited by greater toxicity and rates of local control that are at best, comparable to ${ }^{125} \mathrm{I}$ seed therapy. However, PRS is notable for excellent rates of regional control and greater control of radioresistant lesions than external radiosurgery [8]. Though PRS suffers from potentially use limiting issues of toxicity like ${ }^{125}$ I seed BT, its excellent rates of regional control may warrant further investigation in the treatment of brain metastases. The rates of radiation necrosis are comparable to ${ }^{125}$ I seed brachytherapy, with higher rates of postoperative complications [8,9]. Another major limitation of PRS is that the device used in many of the clinical studies is no longer commercially available. The field awaits the development of another intraoperative or electronic brachytherapy device specialized in intracranial applications [33].

The most recent development in brain brachytherapy is the use of the ${ }^{131} \mathrm{Cs}$ isotope. This isotope shows promising results regarding toxicity, which did not permit brachytherapy to be commonly used for treatment of brain metastases, namely high rates of radiation necrosis and post-operative complications. Studies by Wernicke and colleagues on ${ }^{131} \mathrm{Cs}$ seed implantation, preceded by surgical resection of tumor, are significant for no cases of radiation necrosis and limited post-operative complications related to ${ }^{125}$ I seed implantation $[10,11,12,13]$. These results, especially the lack of radiation necrosis in ${ }^{131} \mathrm{Cs}$ as compared to ${ }^{125} \mathrm{I}$, can be partially explained by several radiobiological advantages of ${ }^{131} \mathrm{Cs}$ over ${ }^{125} \mathrm{I}$. Firstly, ${ }^{131} \mathrm{Cs}$ has a higher median energy, enabling the use of fewer seeds in a given tumor volume. In addition, it has a higher dose-rate, thereby limiting radiation exposure by allowing delivery of greater proportion of dose in a short time. ${ }^{131} \mathrm{Cs}$ 's shorter half-life further limits the duration of patient's exposure to radiation [11]. Relatively low radiation necrosis rates in ${ }^{131}$ Cs may also be explained by high quality of neurological technique or planning methods, as all these studies were done by Wernicke and colleagues. For instance, low seed activity combined with low ra- diation dose would cause minimize radiation necrosis, so the treatment was planned accordingly [10]. Studies with the use of ${ }^{125}$ I have been done by a wide variety of groups, hence the quality of technique or planning methods may not be as high.

One final reason for the lower rate of radionecrosis in the ${ }^{131} \mathrm{Cs}$ data compared to ${ }^{125} \mathrm{I}$ may simply be the lower biological equivalent dose delivered to normal tissue. A comparison of doses was difficult in the past because of uncertainties in estimating the equivalent prescription between the isotopes based on linear quadratic equation (LQE) and biological equivalent dose (BED) formalism. In 2014, Luo et al. published conversion factors between ${ }^{125} \mathrm{I}$ and ${ }^{131} \mathrm{Cs}$ prescription doses, with a resensitization correction for fast and slow growing tissues [39]. Therefore, the Petr study, which used ${ }^{125} \mathrm{I}$ implants with a prescription dose of $150 \mathrm{~Gy}$ at $5 \mathrm{~mm}$, and which resulted in high radionecrosis rates, would be biologically equivalent to a ${ }^{131} \mathrm{Cs}$ equivalent dose of $110 \mathrm{~Gy}$ for tumor $(\alpha / \beta$ ratio of 10) and a ${ }^{131} \mathrm{Cs}$ equivalent dose of $149 \mathrm{~Gy}$ for normal tissue ( $\alpha / \beta$ ratio of 3 ) [30]. This is a biological equivalent dose that is considerably higher than the $80 \mathrm{~Gy}{ }^{131} \mathrm{Cs}$ dose at $5 \mathrm{~mm}$ that is typically prescribed today. Huang et al. used ${ }^{125} \mathrm{I}$ with a dose of $200 \mathrm{~Gy}$ at $1 \mathrm{~cm}$ from the cavity, and also reported a high radionecrosis rate of $26 \%$ [28]. Other ${ }^{125}$ I studies, which used lower prescription doses in the range of $50-60 \mathrm{~Gy}\left({ }^{131} \mathrm{Cs}\right.$ equivalent doses of 40-50 Gy for normal tissue) reported low rates of radionecrosis $[21,22,23,29]$. Lower equivalent doses used in ${ }^{131} \mathrm{Cs}$ brachytherapy appear to result in similar local control to high-dose ${ }^{125} \mathrm{I}$ while limiting toxicity. Therefore, radiobiologic knowledge of low-dose-rate brachytherapy is important for understanding the risk of toxicity of brain brachytherapy implants.

In addition to decreasing toxicity, ${ }^{131} \mathrm{Cs}$ brachytherapy may improve quality of life as measured by FACT-BR questionnaire and mini-mental status exam [12]. Recent studies on ${ }^{131} \mathrm{Cs}$ have achieved up to $100 \%$ of local control, durable regional and distant control of disease resistant to SRS and WBRT $[10,11,12,13]$. The ability of ${ }^{131} \mathrm{Cs}$ brachytherapy to accomplish excellent control of disease with limited toxicities, especially compared to therapies such as SRS and WBRT, support the use of brachytherapy as a more conventional treatment for brain metastases [11]. ${ }^{131}$ Cs brachytherapy may also result in improvement in quality of life as measured by FACT-BR questionnaire and the mini-mental status exam [12].

Considering the present state of brachytherapy and all available modalities used to treat brain metastases, ${ }^{131}$ Cs brachytherapy shows a significant promise. Both ${ }^{125} \mathrm{I}$ and ${ }^{131} \mathrm{Cs}$ brachytherapy are notable for excellent rates of both local and regional control, with ${ }^{131}$ Cs possessing ideal radiobiological properties and with possible improvements in radiation necrosis as compared to ${ }^{125} \mathrm{I}$ brachytherapy as well as quality of life $[10,11,12,13]$. This reduction of toxicity may support wider implementation of brachytherapy as a therapy for patients with brain metastases, particularly for those with large or recurrent tumors. Furthermore, it has low rates of radiation necrosis and other post-operative complications. It should be noted 
that there were no studies that met our eligibility criteria that utilized high-dose-rate brachytherapy with ${ }^{192} \mathrm{Ir}$.

Reasons that currently limit the use of brachytherapy are as follows: 1) The status of brachytherapy as an invasive procedure necessitating hospitalization; 2) The absence of radiation oncologists' or neurosurgeons' expertise in brachytherapy; 3) The lack of published data on treatment outcomes; 4) The increasing role of stereotactic radiosurgery, which is a minimally invasive procedure used to treat many of the same tumors that can be treated with brachytherapy. Even with these limitations, brachytherapy is well suited for treatment of brain metastases, through its ability to deliver a highdose of radiation confined to the resection cavity, while sparing adjacent radiosensitive tissues. This precision achieved by brachytherapy results in excellent rates of local control and improved quality of life.

\section{Conclusions}

The studies examining brachytherapy in the management of brain metastases are predominantly single center studies, with inconsistencies in reporting, quality control, and choice of isotope. However, the results indicate that brachytherapy warrants further consideration in the management of brain metastases, especially in the setting of recurrent tumors after an initial course of radiation therapy. In addition, more studies must be completed to evaluate brachytherapy as a widely used and accepted method of treatment for brain metastases.

\section{Disclosure}

The authors report no conflict of interest.

\section{References}

1. Mehta MP, Tsao MN, Whelan TJ et al. The American Society for Radiation Oncology (ASTRO) evidence-based review of the role of radiosurgery for brain metastases. Int $\mathrm{J}$ Radiat Oncol Biol Phys 2005; 63: 37-46.

2. Patchell RA. The management of brain metastases. Cancer Treat Rev 2003; 7372: 533-540.

3. Sperduto PW, Kased N, Roberge D et al. Summary report on the graded prognostic assessment: An accurate and facile diagnosis-specific tool to estimate survival for patients with brain metastases. J Clin Oncol 2012; 30: 419-425.

4. Aoyama $\mathrm{H}$, Shirato $\mathrm{H}$, Tago $\mathrm{M}$ et al. Stereotactic radiosurgery plus whole-brain radiation therapy vs stereotactic radiosurgery alone for treatment of brain metastases: A randomized controlled trial. J Am Med Assoc 2006; 295: 2483-2491.

5. Suh JH, Videtic GM, Aref AM et al. ACR Appropriateness

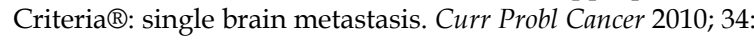
162-174.

6. Lodge WO. Treatment of intrasellar tumours by radon. $\mathrm{Br}$ Med J 1936; 2: 1257-1258.

7. Prados M, Leibel S, Barnett CM et al. Interstitial brachytherapy for metastatic brain tumors. Cancer 1989; 63: 657-660.

8. McDermott MW, Cosgrove GR, Larson DA et al. Interstitial brachytherapy for intracranial metastases. Neurosurg Clin N Am 1996; 7: 485-495.

9. Curry WT, Cosgrove GR, Hochberg FH et al. Stereotactic interstitial radiosurgery for cerebral metastases. J Neurosurg 2005; 103: 630-635.
10. Wernicke AG, Yondorf MZ, Peng L et al. Phase I/II study of resection and intraoperative cesium-131 radioisotope brachytherapy in patients with newly diagnosed brain metastases. J Neurosurg 2014; 121: 338-348.

11. Wernicke AG, Smith AW, Taube $S$ et al. Cesium-131 brachytherapy for recurrent brain metastases: durable salvage treatment for previously irradiated metastatic disease. J Neurosurg 2017; 126: 1212-1219.

12. Pham A, Yondorf MZ, Parashar B et al. Neurocognitive function and quality of life in patients with newly diagnosed brain metastasis after treatment with intra-operative cesium-131 brachytherapy: a prospective trial. J Neurooncol 2016; 127: 63-71.

13. Wernicke AG, Hirschfeld CB, Smith AW et al. Clinical outcomes of large brain metastases treated with neurosurgical resection and intraoperative cesium-131 brachytherapy: results of a prospective trial. Int J Radiat Oncol Biol Phys 2017; 98: 1059-1068.

14. Liberati A, Altman DG, Tetzlaff J et al. The PRISMA statement for reporting systematic reviews and meta-analyses of studies that evaluate health care interventions: explanation and elaboration. J Clin Epidemiol 2009; 62: e1-34.

15. Pouliot J, Beaulieu L. Modern Principles of Brachytherapy Physics: From 2-D to 3-D to Dynamic Planning and Delivery. In Hoppe RT, Philips TL, Roach M (eds.). Leibel and Phillips Textbook of Radiation Oncology (3th ed.). Elsevier Inc., Philadelphia, PA 2010; 224-244.

16. Zlokazov S, Swanberg DJ, Egorov O et al. Method for largescale production of Cesium-131 with low Cesium-132 content. United States Pat Appl 2012; 1-4.

17. Alesch F, Hawliczek R, Koos WT. Interstitial irradiation of brain metastases. Acta Neurochir Suppl 1995; 63: 29-34.

18. Romagna A, Schwartz C, Egensperger R et al. Iodine- 125 brachytherapy as upfront and salvage treatment for brain metastases. Strahlenther Onkol 2016; 192: 780-788.

19. Schulder M, Black PML, Shrieve DC et al. Permanent low-activity iodine-125 implants for cerebral metastases. J Neurooncol 1997; 33: 213-221.

20. Teixeira MJ, Lepski G, Correia C et al. Interstitial irradiation for CNS lesions, in stereotactic and functional neurosurgery. Stereotact Funct Neurosurg 2003; 81: 24-29.

21. Ruge MI, Kickingereder P, Grau $S$ et al. Stereotactic biopsy combined with stereotactic 125iodine brachytherapy for diagnosis and treatment of locally recurrent single brain metastases. J Neurooncol 2011; 105: 109-118.

22. Ruge MI, Suchorska B, Maarouf M et al. Stereotactic 125Iodine brachytherapy for the treatment of singular brain metastases: Closing a gap? Neurosurgery 2011; 68: 1209-1218.

23. Ruge MI, Kocher M, Maarouf $M$ et al. Comparison of stereotactic brachytherapy (125Iodine Seeds) with stereotactic radiosurgery (LINAC) for the treatment of singular cerebral metastases. Strahlentherapie Onkol 2011; 187: 7-14.

24. Zamorano L, Yakar D, Dujovny M et al. Permanent iodine-125 implant and external beam radiation therapy for the treatment of malignant brain tumors. Stereotact Funct Neurosurg 1992; 59: 183-192.

25. Bernstein M, Cabantog A, Laperriere N et al. Brachytherapy for recurrent single brain metastasis. Can J Neurol Sci 1995; 22: $13-16$.

26. Bogart JA, Ungureanu C, Shihadeh E et al. Resection and permanent I-125 brachytherapy without whole brain irradiation for solitary brain metastasis from non-small cell lung carcinoma. J Neurooncol 1999; 44: 53-57.

27. Dagnew E, Kanski J, McDermott MW et al. Management of newly diagnosed single brain metastasis using resection and permanent iodine-125 seeds without initial whole-brain radiotherapy: a two-institution experience. Neurosurg Focus 2007; 22: 20-25. 
28. Huang K, Sneed PK, Kunwar S et al. Surgical resection and permanent iodine-125 brachytherapy for brain metastases. J Neurooncol 2009; 91: 83-93.

29. Ostertag $C B$, Kreth FW. Interstitial iodine- 125 radiosurgery for cerebral metastases. Br J Neurosurg 1995; 9: 593-604.

30. Petr MJ, McPherson CM, Breneman JC et al. Management of newly diagnosed single brain metastasis with surgical resection and permanent I-125 seeds without upfront whole brain radiotherapy. J Neurooncol 2009; 92: 393-400.

31. Raleigh DR, Seymour ZA, Tomlin B et al. Resection and brain brachytherapy with permanent iodine- 125 sources for brain metastasis. J Neurosurg 2017; 126: 1749-1755.

32. Rogers LR, Rock JP, Sills AK et al. Results of a phase II trial of the GliaSite Radiation Therapy System for the treatment of newly diagnosed, resected single brain metastases. J Neurosurg 2006; 105: 375-384.

33. Dinsmore M, Harte KJ, Sliski AP et al. A new miniature x-ray source for interstitial radiosurgery: device description. Med Phys 1996; 23: 45-52.

34. Brennan C, Yang TJ, Hilden P et al. A phase 2 trial of stereotactic radiosurgery boost after surgical resection for brain metastases. Int J Radiat Oncol Biol Phys 2014; 88: 130-136.

35. Jagannathan J, Yen CP, Ray DK et al. Gamma Knife radiosurgery to the surgical cavity following resection of brain metastases. J Neurosurg 2009; 111: 431-438.

36. Chang EL, Wefel JS, Hess KR et al. Neurocognition in patients with brain metastases treated with radiosurgery or radiosurgery plus whole-brain irradiation: a randomised controlled trial. Lancet Oncol 2009; 10: 1037-1044.

37. Murray KJ, Scott C, Zachariah B et al. Importance of the mini-mental status examination in the treatment of patients with brain metastases: A report from the radiation therapy oncology group protocol 91-04. Int J Radiat Oncol Biol Phys 2000; 48: 59-64.

38. Regine WF, Schmitt FA, Scott CB et al. Feasibility of neurocognitive outcome evaluations in patients with brain metastases in a multi-institutional cooperative group setting: Results of Radiation Therapy Oncology Group trial BR-0018. Int J Radiat Oncol Biol Phys 2004; 58: 1346-1352.

39. Luo W, Molloy J, Aryal P et al. Determination of prescription dose for Cs-131 permanent implants using the BED formalism including resensitization correction. Med Phys 2014; 41: $1-8$. 